\title{
SOLAR SPECTROPOLARIMETER FOR SPACE WEATHER FORECAST
}

\section{E.F. Ivanov}

Institute of Solar-Terrestrial Physics SB RAS, Irkutsk,Russia,eugenessrt@gmail.com

\section{A.V. Gubin}

Institute of Solar-Terrestrial Physics SB RAS, Irkutsk,Russia,gubin@iszf.irk.ru

\author{
S.V. Lesovoi \\ Institute of Solar-Terrestrial Physics SB RAS, \\ Irkutsk,Russia,svlesovoi@gmail.com \\ Ramses Zaldivar Estrada \\ Institute of Geophysics and Astronomy, Cuban Academy of Sciences, \\ Havana,Cuba,ramses@iga.cu
}

struments at various longitudes will allow continuous observation of type II bursts, often related to coronal mass ejections (CMEs) - the most geoeffective solar activity events that affect the space weather significantly.

Keywords: space weather, solar radio emission, dynamic spectrum. ling and transmitting data remotely via the Internet Along with these requirements, the proposed SDR solution allows us to measure Stokes I and V easily, which contrasts the proposed instrument with e-CALLISTO network spectropolarimeters, most of which can record only one linear polarization. Deployment of such in-

\section{INTRODUCTION}

Development of communication and global positioning systems, as well as geospace exploration in recent decades have led to the need for diagnostics and prediction of geospace conditions. The determining factor responsible for geospace conditions is solar activity. The entire complex of factors associated with the effect of solar activity on geospace and directly on Earth is called space weather (SW). One of the objectives of the $\mathrm{SW}$ forecast is to record coronal mass ejections (CMEs) - the most geoeffective events, i.e. solar activity effects causing distortions of magnetic and radiation situations in geospace and different atmospheric, ionospheric, and magnetospheric layers. Characteristic examples of consequences of such events are radio failure, rise in the radiation level, magnetic storms.

SW is most strongly affected by CMEs. Along with optical coronagraphs such as LASCO [Brueckner et al., 1995], these events are often recorded in the meter wavelength range of solar radio emission. CMEs can cause shock waves in coronal plasma. In turn, shocks propagating in the outer solar corona can generate meter bursts slowly drifting downward in frequency [Grechnev et al., 2011, Cho et al., 2013]. The circular polarization degree of type II bursts is usually low, but the circular polarization degree of herringbone structures of type II burst may run to $80 \%$ [Dorovskyy et al., 2015].

As shown by the experience of processing of data from e-CALLISTO network spectropolarimeters, most of which can record only one linear polarization, responses of different instruments to the same event differ. It is difficult to determine the cause of this difference - different characteristics of receivers or ionospheric effect. To minimize ambiguities in the interpretation of data from the network of instruments, they should be as more identical as possible and measure Stokes $I$ and $V$.

Receivers are the most identical with maximum use of digital signal processing. It is more reasonable to perform all operations on a signal, except for preamplification, in digital form.

The main subject of the study of the spectropolarimeter under design is type II bursts as indicators of CMEs. Such solar radio bursts are recorded in a range of relatively low frequencies to $1 \mathrm{GHz}$, which allows digital signal processing without the use of expensive microwave (MW) components and high-speed analogto-digital converters (ADCs). In this case, the analog part of the receiver can be simplified to the utmost: antenna, preamplifier, and, if necessary, anti-aliasing filter. Mass-produced SDR devices are perfectly suited for these purposes. In the military field, SDR solutions have been used since the early 2000s [Dillinger et al., 2003], but in fact SDR devices became freely available on cost and applicability only during the last three-five years due to the development of broadband wireless communication standards, as was in the case of solutions based on field-programmable gate arrays (FPGA).

Classification and early detection of geoeffective events require a global network of spectropolarimeters combining qualities such as flexibility of configuration depending on the interference situation, low cost, ease of deployment and maintenance of spectropolarimeter systems. We think that all these requirements are met by the signal processing systems based on SDR solutions [Akeela, Dezfouli, 2018]. Software-configured technologies allow us to transfer most of the receiver to the digital domain, hence radio frequency parameters of the receiver are defined by software. An ideal SDR device should convert a signal at the antenna output into a digi- 
tal form and conduct all further operations through software. It is, however, impossible to do without a low noise amplifier that transforms a signal into the range of ADC input signals.

Real SDR devices include an amplifier, a synthesizer, and a mixer. A signal is digitized at an intermediate frequency. All other operations - filtering, demodulation, implementation of protocols — are carried out through software. Synthesizers are also controlled by software, therefore the operating frequency, which is important for the proposed project, is selected in software too. SDR solutions are still relatively rare in radio astronomy, and amateur astronomers were the first who used them [https://www.rtl-sdr.com/category/ radio-astronomy-2]. Recently, however, there has appeared information about the use of SDR in major radio astronomy projects [Sabater, Johnston, 2019] and about SDR courses in university programs.

Of particular importance for SW forecast is the continuous monitoring of solar activity. It is possible by installing spectropolarimeters in orbital observatories (STEREO [https://swaves.gsfc.nasa.gov/swaves instr.html]), or at different longitudes (e-CALLISTO [http://ecallisto.org]), or in polar regions. In this paper, we propose a project of spectropolarimeter for the 70$500 \mathrm{MHz}$ frequency range to be deployed in the ground network at different longitudes. Besides Russia, such spectropolarimeters are planned to be installed in the Havana Radio Astronomy Station [http://www.gaoran.ru/russian/publs/information/gavana. pdf]. If the spectropolarimeters are placed at the most easterly and westerly points of Russia (PetropavlovskKamchatsky, Kaliningrad) and in Cuba, we will get an almost complete observation overlap with a longitude separation of $\sim 120^{\circ}$.

While the main type of bursts of interest for SW forecast is type II, this instrument is designed to record radio bursts of any types. Consequently, when designing the receiver, we should take into account all characteristic spectral, temporal, polarization features of bursts of known types. The events that require the highest temporal resolution are millisecond radio spikes - a type of the superfine structure of solar flare radio emission, which also comprises pulsations (regular and irregular) with a characteristic time of less than $1 \mathrm{~s}$ [Fleishman, Melnikov, 1998]. The temporal resolution of $10 \mathrm{~ms}$ is sufficient for reliable recording.

The spectral resolution of the future instrument should be sufficient for reliable recording of bursts with fine spectral structure. For example, to identify zebra pattern structures - drifting periodic narrowband structures in the dynamic spectrum against a long-term type IV burst [Chernov, 2006] — requires a $1 \%$ spectral resolution of the full frequency range. On the other hand, in the frequency switching mode the time of scanning of the entire band depends on the number of frequency channels. It therefore seems unreasonable to make the spectral resolution lower than 1 $\%$ for SW forecast.

Observations of the linear polarization in meter wavelengths are difficult because of the strong depo- larization of emission in the frequency range due to the Faraday effect in the solar corona and Earth's ionosphere. The spectropolarimeter will therefore measure the circular polarization up to at least $5 \%$.

\section{METER WAVELENGTH SOLAR RADIO TELESCOPES}

Meter wavelength spectropolarimeters designed to monitor solar activity have been developed since the 1950s [Payne-Scott, Little, 1951]. At the same time, types of observable solar radio bursts were determined and assumptions were made about mechanisms of their generation. Nowadays, in the meter wavelength range the Sun is observed with spectropolarimeters and interferometers.

Ground-based meter wavelength observations can be made only down to $\sim 20 \mathrm{MHz}$, lower frequency radiation does not penetrate through Earth's ionosphere. The lowfrequency range is studied using spectropolarimeters installed in spacecraft such as WIND and STEREO [Bougeret et al., 1995, 2008]. Ground-based spectropolarimeters in the Learmonth, Culgoora observatories have successfully operated in respectively $25-180 \mathrm{MHz}$ and $18-1800 \mathrm{MHz}$ frequency ranges for several decades. The ground-based solar spectropolarimeter AMATERAS in Fukushima (Japan) has relatively recently started observations [Iwai et al., 2013]. It measures solar radio emission in the $150-500 \mathrm{MHz}$ frequency range with a temporal resolution of $10 \mathrm{~ms}$ and a frequency resolution of $61 \mathrm{kHz}$ in both circular polarizations simultaneously. The Greek spectrograph ArtemisJLS also works successfully. This spectrograph has a temporal resolution of $10 \mathrm{~ms}$ with 128 frequency channels in the $270-450 \mathrm{MHz}$ frequency range and $100 \mathrm{~ms}$ in the $20-650 \mathrm{MHz}$ frequency range in the mode of sequential switching of operating frequency. Recent works carried out by analyzing data from this spectrograph [Bouratzis et al., 2019] have shown that such instruments can solve fundamental problems of solarterrestrial physics.

The low-frequency interferometers LOFAR and MWA, although not being solar instruments, make solar observations either. MWA is located in Western Australia, the number of its elements is 2048, the frequency range is $80-300 \mathrm{MHz}$, the channel bandwidth is 30.72 $\mathrm{MHz}$, the spectral resolution is $40 \mathrm{kHz}$, the temporal resolution is $0.5 \mathrm{~s}$; it measures all Stokes parameters ( $I$, $Q, U, V)$ [Tingay et al., 2013]. LOFAR is a European radio interferometer with a $10-240 \mathrm{MHz}$ frequency range, $0.7 \mathrm{kHz}$ frequency bandwidth, and $1158 \mathrm{~km}$ maximum base. LOFAR antennas record two orthogonal linear polarizations. Depending on the problem, these polarizations are converted into Stokes $I$ or into the full set of Stokes parameters [van Haarlem et al., 2013].

The International network of spectropolarimeters eCALLISTO [Benz et al., 2005, 2009] is the unique ground-based solar instrument capable of continuously observing the Sun in the meter wavelength range. Data from all instruments of the network is promptly collected on one website and are freely available 
[http://soleil.i4ds.ch/solarradio/callistoQuicklooks]. As noted above, the drawback of e-CALLISTO is that almost all instruments of this network measure only one linear polarization. It also seems inconvenient to interface e-CALLISTO receivers with a computer through a serial port or USB. It would be more preferable to insert the receivers into the local network or connect them to the Internet. Then we can get rid of the mandatory requirement of having a computer in each spectropolarimeter of the network. From the viewpoint of the coverage of different longitudes, the e-CALLISTO network can be considered perfect.

\section{TYPICAL SCHEMES OF METER WAVELENGTH SPECTROPOLARIMETERS}

In the hardware design, state-of-the-art radio instruments recording the spectrum, which meet the formulated conditions, can be conveniently divided into three types.

1. Agile (sequential) instruments. The receive path of such instruments features a narrow bandwidth defining a bandwidth of one frequency channel. The desired number of frequency channels is determined by their sequential switching (sweeping) by tuning frequency of a local oscillator.

Advantages of such instruments are:

- simplicity of implementation (in case of receiving one polarization);

- existence of ready solutions (e.g., frequency synthesizers for television receivers) for a range below 1 $\mathrm{GHz}$;

- use of cheap low-speed ADCs and microcontroller units with low performance for gathering data.

Their disadvantages are:

- complex and bulky analog part of the receive path (amplification, frequency conversion, band forming, image rejection must be implemented in analog form);

- analog path noises and distortions;

- receiving two orthogonal linear polarizations requires developing a two-channel receiver;

- receiving two circular polarizations in addition to the previous requirements adds the need for relatively expensive equipment — antennas receiving circular polarization or a wideband hybrid coupler converting two linear polarizations into circular.

2. Digital (parallel) instruments. In such instruments, the entire frequency band of interest is fed to an analog-todigital converter, and frequency channels are digitally formed by digital signal processing methods (e.g., [Gubin, Lesovoi, 2012]).

Advantages of instruments of this type are:

- simplification and cheapening of the analog part by digitizing many signal processing stages (band forming, image rejection, conversion of circular polarizations from linear ones);

- higher temporal resolution can be achieved through parallel processing of frequency channels;

- more predictable and stable characteristics of the receive path (identity of frequency channels, temperaturedependent stable characteristics of frequency channels);

- flexibility - capability of changing characteristics of the receive path by reprogramming digital signal processing algorithms.

Their disadvantages are:

- cost increase due to more expensive high-speed ADCs as well as due to higher data rate requiring the use of FPGA or digital signal processors (DSPs) of great performance;

- considerable labor input to programming and description of digital signal processing algorithms;

- digital noise.

3. Hybrid-type instruments are a combination of instruments of the two previous types in which the entire frequency band of interest is divided into subbands. Frequency channels are formed within each subband in digital form, switching between the subbands is performed sequentially by tuning an oscillator frequency. The method is used to reach a compromise between instruments of the first and second types under certain conditions to solve a particular problem (e.g., the need to exploit a wider bandwidth than that which can be digitized and processed entirely with available equipment). Depending on the selected configuration, advantages and disadvantages of instruments of the first or second types can prevail.

\section{FEASIBILITY OF APPLICATION OF SDR IN METER WAVELENGTH SPECTROPOLARIMETERS}

At present, the SDR technology is becoming more common in commercial radio systems. It is a transceiver whose main characteristics can be controlled by software. In fact, SDR devices are a product of the evolution of tuners used in consumer radio sets. Such tuners can usually also control the main characteristics: operating frequency, band, and others. A tuner is, however, usually designed for a specific broadcast standard (TV, radio, amateur mobile radio) and ranges of tuning of its characteristics are within the scope of the standard. The main difference of SDR devices is that they are designed as a universal solution to satisfy the needs of various standards. For example, the same SDR device can be configured to work via Bluetooth, WiFi, WCDMA, satellite communications, and many others. This is achieved through a large number of controlled characteristics of the system, large ranges of variation of these characteristics, and a high degree of integration of individual elements of the transceiver path into one chip or one card.

Due to the availability of commercial devices based on the SDR technology, they are beginning to find application in scientific instruments and installations. An example is the ionospheric sounding network [Podlesniy, 2017] as well as the amateur radio astronomy receivers mentioned above. In most of these examples, cheap SDR products (RTL-SDR and the like), designed for use with PC via USB, are applied. A feature of such products is a relatively nar- 
row channel bandwidth and low digital data rate via USB. Currently, however, there are more advanced SDR products being, of course, more expensive, but having greater capabilities. An example of such a product is the AD9361 chip by Analog Devices [https://www.analog.com/en/analog-dialogue/articles/ using-model-based-design-sdr-1.html]. Let us take a brief look at its main characteristics:

1. Two independent receiving channels can be used to receive two linear polarizations for further conversion to Stokes I and V in digital form;

2. Operating frequency: $70 \mathrm{MHz}-6 \mathrm{GHz}$;

3. Channel bandwidth: $200 \mathrm{kHz}-56 \mathrm{MHz}$;

4. Two 12-bit ADCs for each receive channel (for $I$ and $Q$ components);

5. Maximum speed of ADC operation: 640 MSPS;

6. Controlled gain: 0-80 dB;

7. Local oscillator frequency switching step: $2.4 \mathrm{~Hz}$;

8. Built-in $I / Q$ mixers;

9. 128-order built-in FIR filters at ADC outputs;

10. Built-in decimators with variable window functions.

As the base element of the receive path of the spectropolarimeter, we propose to use ARRadio kit by Terasic [https://wiki.analog.com/resources/eval/userguides/ arradio]. The ARRadio kit includes a SDR-transceiver chip AD9361, a source clock signal with up to $\pm 10^{-5}$ stability (with the possibility of using an external source), and a High Speed Mezzanine Card (HSMC) digital interface allowing us to connect ARRadio to development kits Intel (Altera). The cost of the ARRadio kit is comparable with the cost of the wideband hybrid coupler in the finished device, ready to be connected by means of radio frequency connectors, which is another reason for its choice for the spectropolarimeter.

ARRadio can be utilized with virtually any Altera development kit, which comprises the HSMC interface. It is, however, recommended to use the Terasic DE10-Standard development kit to reduce the design time and increase capabilities [https://rocketboards.org/foswiki/ Documentation/DE10Standard]. This kit is based on the FPGA family Cyclone-5, which has a built-in ARM processor enabling us to use the operating system (OS) Linux, for which there are drivers and software for the steep learning curve of ARRadio. The application of such a development kit with the processor and the possibility of using the full-fledged OS allow us to design a stand-alone instrument that does not require $\mathrm{PC}$ in close proximity (Figure).

Using the proposed components, we can construct both an agile spectropolarimeter and a hybrid-type one (covering the full frequency range of the instrument by switching subbands $56 \mathrm{MHz}$ wide). In the former case, the channel switching rate may greatly depend on the frequency switching time. Since the AD9361 transceiver is an integrated unit consisting of a set of blocks, it has a developed system of calibrations of RF paths and internal network of the local oscillator. According to the technical manual for the transceiver [https://www.analog.com/ en/design-center/landing-pages/001/integrated-rf-agiletransceiver-design-resources.html], it takes most time to calibrate the voltage controlled oscillator of the local os- cillator path. It may be as long as $40-140 \mu \mathrm{s}$, depending on the operating mode of the transceiver's receive path. Hence, if we take the temporal resolution of one frequency channel as $1 \mathrm{~ms}(0.5 \mathrm{~s}$ for the entire frequency range 70 $500 \mathrm{MHz}$ with a frequency resolution of $1 \mathrm{MHz}), 5-15 \%$ of single frequency channel measurement time would be spent on calibration. It is feasible, however, if there is a need to increase the temporal resolution up to $10^{-5}-10^{-4} \mathrm{~s}$, the calibration time will be the limiting factor. Thus, to obtain such temporal resolution using the AD9361 transceiver, the only solution is the hybrid-type scheme of the spectropolarimeter.

The question about the possibility of obtaining Stokes $I$ and $V$ from linear polarizations also requires to be addressed. The easiest way to accomplish this objective is to shift the phase of the local oscillator signal in the receive path of one of the linear polarizations. Besides obtaining Stokes parameters, we, however, have to perform another operation associated with the oscillator signal phase shift — image frequency rejection [https://www.analog.com/ru/analog-dialogue/articles/ mirror-mirror-on-the-wall-understanding-image-

rejection-and-its-impact-on-desired-signals.html]. If the AD9361 chip has insufficient capabilities of manipulating the phases to implement simultaneously these two objectives, there is an alternate variant - to obtain Stokes $I$ and $V$ in digital form in FPGA [Das et al., 2010].

One of the main advantages in using the SDR solution to develop a receiver of the spectropolarimeter is flexibility. Many characteristics of such a receiver are easy to change by software-based methods according to the objective or to conduct certain experiments. The frequency range is largely limited only by capabilities of antenna and pre-amplifiers because it is hardly possible to find one antenna and amplifiers for the $0.07-6 \mathrm{GHz}$ range with satisfactory characteristics for radio astronomy purposes.

The maximum temporal resolution of one frequency channel for the agile scheme as described above is restricted to values of the order of hundreds of microseconds. The temporal resolution can be improved in two ways. The first method can be used for a small number of frequency channels. In this case, it is possible to significantly reduce the time of calibration of the SDR-chip receive path by downloading ready-made lists of calibration coefficients for each frequency channel. The temporal resolution in this operating mode will be of the order of tens of microseconds. The second method is realized in the absence of local oscillator frequency switching. The maximum temporal resolution here will be limited only by the chip maximum sampling frequency of 60 MSPS, and therefore a bottleneck defining the receiver's high-speed performance becomes the high-speed performance of blocks of subsequent digital signal processing in FPGA, as well as the rate of data transmission from FPGA to PC or local network.

The maximum frequency resolution when using only SDR-chip resources for band forming, according to the characteristics, is $200 \mathrm{kHz}$. It, however, can also be substantially improved, if necessary, by further separating 


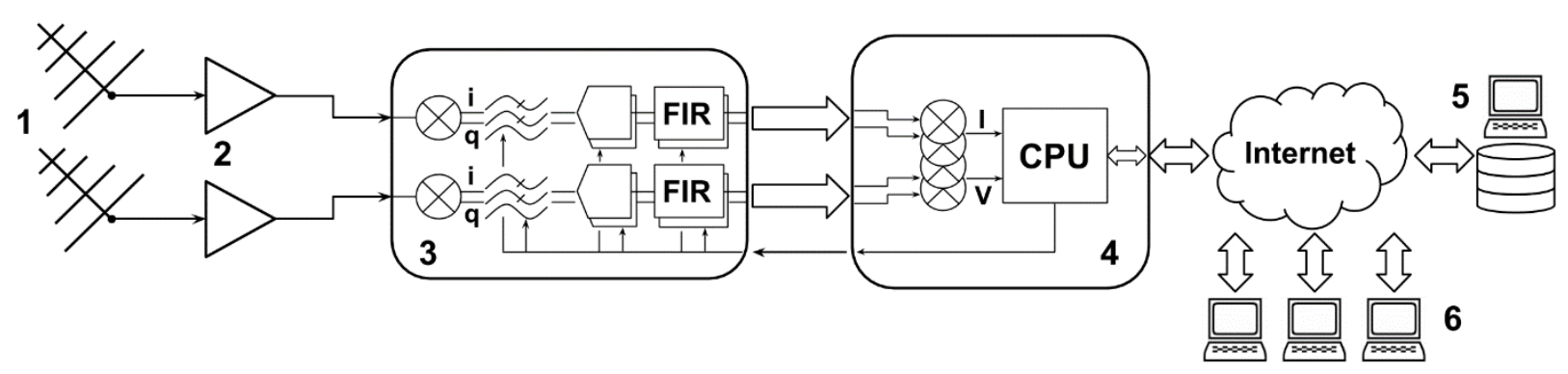

Block diagram of the proposed instrument: 1 - two orthogonal log-periodic antennas; 2 - low noise amplifiers; 3 - daughter card ARRadio; 4 - development kit DE10-Standard; 5 - storage server; 6 - data users; FIR - digital filter; CPU - central processing unit

the band into more frequency channels in digital form in FPGA.

The number of frequency channels, in fact, is not limited and is determined by the ratio of the total operating frequency band of the instrument to the desired band of one channel.

\section{MAIN CHARACTERISTICS OF THE PROPOSED SPECTROPOLARIMETER}

On the basis of the objectives as well as simplicity of implementation, easy maintenance, and reduced need for resources (in particular, for the Internet connection and data storage), we propose developing a spectropolarimeter with the following characteristics:

- full frequency range: $70-500 \mathrm{MHz}$;

- frequency resolution: $1 \mathrm{MHz}$;

- temporal resolution: $0.5 \mathrm{~s}$;

- number of frequency channels: 430 ;

- recording of Stokes $I$ and $V$.

The work with a SDR receiver suggests using two orthogonal log-periodic antennas operating in a desired frequency range. In addition, to improve the sensitivity it is suggested to use two low-noise amplifiers - one for each antenna.

According to preliminary estimate, the amount of data for the instrument with the above parameters will be about $300 \mathrm{MB} /$ day (approximately $100 \mathrm{~GB} /$ year). For reliable transfer of such amount of data it suffices to connect to the Internet at a speed of $128 \mathrm{kbit} / \mathrm{s}$.

Precise timing can be carried out in two ways. To simplify and cheapen the spectropolarimeter, we can synchronize it via the Internet: modern NTP versions can provide timing accuracy up to $10 \mathrm{~ms}$ [http://www.ntp.org/ ntpfaq/NTP-s-algo.htm]. If there is a need for a more precise timing or stability during short interruptions in connection to the Internet, the spectropolarimeter may be equipped with a GPS receiver.

\section{CONCLUSION}

The proposed version of the solar spectropolarimeter is optimal in terms of costs and functionality. The use of commercially available SDR devices along with devices containing FPGA allows us to develop inexpensive, identical spectropolarimeters with remote access to data and possibility of remote reconfiguration for certain tasks. The spectropolarimeter parameters - frequency band, number of frequency channels, number of polarizations - are quite suitable for recording solar activity in the meter wavelength range. The application of spectropolarimeters of this type in the network (deployed at different longitudes) to space weather forecast seems quite rational: they do not require maintenance - they need only main power feed and Internet connection.

We believe that the described engineering proposals for the development of a meter wavelength spectropolarimeter can be a useful aid to developing the Russian (and in the future to improving the International) network of meter wavelength range solar spectropolarimeters.

This work was performed with budgetary funding of the Russian Foundation for Basic Research 18-52-34004 and SITMA under the research project "Upgrading of the Havana radio astronomy station for monitoring solar activity and fundamental solar research", RAS Presidium program No. 56 "Fundamentals of innovative national security technologies", and Basic Research program 16.II.

\section{REFERENCES}

Akeela R., Dezfouli B. Software-defined Radios: Architecture, State-of-the-art, and Challenges, 2018 // Arxiv. URL: https://arxiv.org/abs/1804.06564 (accessed 30 September 2019).

Benz A.O., Monstein C, Meyer H. Callisto A new concept for solar radio spectrometers. Solar Phys. 2005, vol. 226, iss. 1, pp. 143-151.

Benz A.O., Monstein C., Meyer H., et al. //Earth Moon and Planets. 2009. V.104, iss. 1-4. P. 277-285 DOI: 10.1007/s11038-008-9267-6.

Bougeret J.-L., Kaiser M.L., Kellogg P.J., Manning R., Goetz K., Monson S.J., Monge N., Friel L., Meetre C.A., Perche C., Sitruk L., Hoang S. Waves: the radio and plasma wave investigation on the Wind spacecraft. Space Sci. Rev. 1995, vol. 71, iss. 1-4, pp. 231-263.

Bougeret J.L., Goetz K., Kaiser M.L., Bale S.D., Kellogg P.J., Maksimovic M., et al. S/WAVES: the radio and plasma wave investigation on the STEREO Mission. Space Sci. Rev. 2008, vol. 136 , iss. $1-4$, pp. $487-528$.

Bouratzis C., Hillaris A., Alissandrakis C.E., PrekaPapadema P., Moussas X., Caroubalos C., Tsitsipis P., Kontogeorgos A. Astron. Astrophys. 2019, vol. 625, A58, DOI: 10.1051/0004-6361/201834792.

Brueckner, G. E., Howard, R. A., Koomen, M. J., et al. The Large Angle Spectroscopic Coronagraph (LASCO). Solar Physics. 1995, Volume 162, Issue 1-2, pp. 357-402 DOI: 


\subsection{7/BF00733434}

Chernov G.P. Solar radio bursts with drifting stripes in emission and absorption. Space Sci. Rev. 2006, vol. 127, pp. 195-326.

Cho K.-S., Gopalswamy N., Kwon R.-Y., Kim R.-S., Yashiro S. A high-frequency type II solar radio burst associated with the 2011 February 13 coronal mass ejection. Astrophys. J. 2013, vol. 765 , no. 148,9 p. DOI: $10.1088 / 0004-$ 637X/765/2/148.

Das K., Roy A.L., Keller R., Tuccari G. Conversion from linear to circular polarization in FPGA. Astron. Astrophys. 2010, vol. 509, 11 p. DOI: 10.1051/0004-6361/200913212.

Dillinger M., Madani K., Alonistioti N. Software defined radio: architectures, systems, and functions. John Wiley \& Sons Ltd, 2003. 456 p.

Dorovskyy V.V., Melnik V.N., Konovalenko A.A., Brazhenko A.I., Panchenko M., Poedts S., Mykhaylov V.A. Fine and superfine structure of decameter-hectometer type II burst on 2011 June 7. Solar Phys. 2015, vol. 290, iss. 7, pp. 20312042. DOI: 10.1007/s11207-015-0725-9.

Fleishman G.D., Melnikov V.F. Millisecond solar radio spikes. Uspekhi fizicheskikh nauk [Physics-Uspekhi]. 1998. vol. 41 , no. 12 , pp. 1157-1189. DOI: 10.1070/PU1998v041 n12ABEH000510.

Grechnev V.V., Uralov A.M., Chertok I.M., Kuzmenko I.V., Afanasyev A.N., Meshalkina N.S., Kalashnikov S.S., Kubo Y. Coronal shock waves, EUV waves, and their relation to CMEs. I. Reconciliation of "EIT waves", type II radio bursts, and leading edges of CMEs. Solar Phys. 2011, vol. 273, iss. 2, pp. 433-460. DOI: $10.1007 / \mathrm{s} 11207-011-9780-\mathrm{z}$.

Gubin A.V., Lesovoi S.V. SSRT digital broadband correlator. Vestnik IrGTU [Proc. of Irkutsk State Technical University]. 2012, no. 1, p. 132. (In Russian).

Iwai K., Tsuchiya F., Morioka A., Misawa H. IPRT/AMATERAS: a new metric spectrum observation system for solar radio bursts. Solar Phys. 2013, vol. 277, iss. 2, pp. 447-457.

Payne-Scott R., Little A.G. The position and movement on the solar disk of sources of radiation at a frequency of $97 \mathrm{Mc} / \mathrm{s}$. II. Noise storms. Australian J. Sci. Res. A. 1951, vol. 4, p. 508.

Podlesnyi A.V. A SDR chirp receiver. Mezhdunarodnaya Baikal'skaya molodezhnaya nauchnaya shkola po fundamental'noi fizike [Baikal Young Scientists' International School on Fundamental Physics]. 2017, pp. 200-2002. (In Russian).

Sabater J., Johnston S. Highlights on Spanish Astrophysics X. Proc. XIII Scientific Meeting of the Spanish Astronomical Society. July 16-20, 2018, Salamanca, Spain. 2019, pp. 663-663.
Tingay S.J., Goeke R., Bowman J.D., Emrich D., Ord S.M., Mitchell D.A., et al. The Murchison widefield array: the square kilometre array precursor at low radio frequencies. Publ. of the Astron. Soc. of Australia. 2013, vol. 30, id. e007, 21 p.

van Haarlem M.P., Wise M.W., Gunst A.W., Heald G., McKean J.P., Hessels J.W.T. LOFAR: the LOw-Frequency Array. Astron. Astrophys. 2013, vol. 556, id.A2, 53 p.

URL: https://www.rtl-sdr.com/category/radio-astronomy-2 (accessed 30 September 2019).

URL: https://swaves.gsfc.nasa.gov/swaves_instr.html (accessed 30 September 2019).

URL: http://e-callisto.org (accessed 30 September 2019).

URL: http://www.gaoran.ru/russian/publ-s/information/ gavana.pdf (accessed 30 September 2019).

URL: http://soleil.i4ds.ch/solarradio/callistoQuicklooks (accessed 30 September 2019).

URL: https://www.analog.com/en/analog-dialogue/articles/ using-model-based-design-sdr-1.html (accessed 30 September 2019).

URL: https://wiki.analog.com/resources/eval/userguides/ arradio (accessed 30 September 2019).

URL: https://rocketboards.org/foswiki/Documentation/DE10 Standard (accessed 30 September 2019)

URL: https://www.analog.com/en/design-center/landingpages/001/integrated-rf-agile-transceiver-design-resources.html (accessed 30 September 2019).

URL: https://www.analog.com/ru/analog-dialogue/articles/ mirror-mirror-on-the-wall-understanding-image-rejection-and-itsimpact-on-desired-signals.html (accessed 30 September 2019).

URL: http://www.ntp.org/ntpfaq/NTP-s-algo.htm (accessed 30 September 2019).

How to cite this article

Ivanov E.F., Gubin A.V., Lesovoi S.V., Ramses Zaldivar Estrada. Solar spectropolarimeter for space weather forecast. Solar-Terrestrial Physics. 2019. Vol. 5. Iss. 4. P. 21-26. DOI: 10.12737/stp-54201903. 